\title{
Validación de la «escala de competencia parental percibida versión padres (ECPP-P)» para el cuidado de los hijos
}

\author{
Vera-Vásquez Ceci ${ }^{1 a}$, Zaragoza-Tafur Alexandra Rosario ${ }^{1 b}$, Musayón-Oblitas Flor ${ }^{2}$
}

\section{RESUMEN}

La competencia parental es la capacidad práctica que tienen los padres para cuidar, proteger y educar a sus hijos, y las habilidades que poseen para dar una respuesta adecuada y apropiada a sus necesidades para así asegurarles un desarrollo suficientemente sano. Objetivo: determinar la validez del instrumento escala de competencia parental percibida versión padres para medir la competencia parental en el cuidado de sus hijos, en los padres de escolares de $1 .^{\circ}$ a $6 .^{\circ}$ grado de primaria. Material y métodos: se realizó un estudio descriptivo de corte transversal, la escala fue aplicada a 420 padres de escolares de primaria en un colegio nacional de Chacas, Asunción - Ancash, Perú. Se utilizó estadística descriptiva, análisis factorial, y pruebas para analizar la validez. Resultados: la escala presenta valores altos de confiabilidad y una buena adecuación a los datos, lo cual indica que es confiable y válida. A partir del análisis factorial con el método de extracción se propone un nuevo modelo conformado por tres dimensiones, el que posee alta confiabilidad y validez. Conclusiones: la escala competencia parental percibida versión padres constituida por 22 ítems, posee propiedades psicométricas adecuadas, por lo que se le considera un instrumento válido para evaluar el constructo «competencia parental».

Palabras clave: competencia parental, capacidad y habilidad parental, validación. Perú. (Fuente DeCs BIREME).

\section{Validation of the «Parental Perceived Competence Scale Version parents (CPE-P)» for child care}

\begin{abstract}
Parental competence is the practical ability of parents to care for, protect and educate their children, and the skills you possess to give an adequate and appropriate response to their needs and assure a sufficiently healthy development. Objetive: To determine the validity of the instrument scale version parents perceived parental competence to measure parental competence in caring for their children, parents of school from 1st to 6th grade. Material and methods: A descriptive cross-sectional study was performed; the scale was administered to 420 parents of primary school children in a national association of Chacas, Asunción - Ancash, Peru. Descriptive statistics, factor analysis, and testing to analyze the validity was used. Results: The scale shows high values of reliability and a good fit to the data, indicating that it is reliable and valid. From the factor analysis extraction method with a new model consists of three dimensions, which has high reliability and validity is proposed. Conclusions: Perceived parental competence scale version consists of 22 items parents, has adequate psychometric properties, so it is considered a valid tool for assessing the construct «parental competence.».
\end{abstract}

Key words: parental competence, parental capacity and skill, validation. Peru. (Source DeCs BIREME).

\footnotetext{
Licenciada de Enfermería - Clínica Internacional.

Egresada de la Facultad de Enfermería de la Universidad Peruana Cayetano Heredia

Doctora en Salud Pública, profesora principal de la Universidad Peruana Cayetano Heredia
} 


\section{INTRODUCCIÓN}

La validación de un instrumento considera las propiedades psicométricas, estas propiedades se refieren a la capacidad del ítem para diferenciar, distinguir y discriminar entre sujetos que presentan diferentes niveles o grados en el constructo medido por la puntuación de la prueba; por lo tanto, la validez se refiere al grado en que un instrumento mide la variable que pretende medir (1). La validez se obtiene por diferentes tipos de evidencia: evidencia relacionada con el contenido, el criterio y el constructo. La validez de contenido se refiere al grado en que la medición representa al concepto medido, esta se estableció con la evaluación de expertos y la prueba binomial aplicada a los resultados; la validez de constructo es el grado en que el instrumento representa y mide un concepto teórico, se evaluó con el análisis factorial, y mediante el método de extracción se propuso un nuevo modelo (2). La estructura del instrumento escala competencia parental percibida versión padres (ECPP-p), permite evaluar el constructo «competencia parental» mediante cinco dimensiones: «implicación escolar», «dedicación personal», «ocio compartido», «asesoramiento y la orientación» y «asunción del rol de ser padre o madre»; para su estimación se presenta un cuestionario de 22 preguntas con 4 alternativas de respuesta. Se validó la ECPP-p puesto que se requería contar con un instrumento capaz de identificar el modelo de crianza que han desarrollado los padres y para obtener, en un futuro, diagnósticos concretos; además, poder plantear estrategias de intervención de carácter preventivo, así como diseñar líneas futuras de investigación.

La competencia parental engloba dos componentes: capacidades y habilidades parentales; se llama competencias parentales al conjunto de las capacidades prácticas que tienen los padres para cuidar, proteger y educar a sus hijos, asegurándoles un desarrollo suficientemente sano y bajo patrones aprobados por la sociedad; esto permite a los padres afrontar su rol de modo flexible y adaptativo (3). Las capacidades parentales se conforman a partir de la articulación de factores biológicos, hereditarios y su interacción con las experiencias vitales y el contexto sociocultural de desarrollo de los progenitores o cuidadores de un niño, entre estas tenemos; la capacidad de apego, referido a los recursos emotivos, cognitivos y conductuales que tienen los padres o cuidadores para acercarse a sus hijos y responder a sus necesidades (4). Las habilidades parentales permiten dar una respuesta adecuada y apropiada a la necesidad de sus hijos, está en relación con la función nutriente que se relaciona con las experiencias sensoriales y emocionales que permiten construir un ape- go seguro que se vincula entre los niños y sus padres, dedicándoles el tiempo y espacio para conversar, trasmitir valores y aclarar dudas con la finalidad de percibir el mundo familiar como un espacio seguro (5).

Un factor protector en la prevención de problemas sociales es el desarrollo de una competencia parental positiva, desarrollar una estabilidad emocional y brindar a los hijos apoyo escolar, calidez, afecto y confianza, puesto que mejoran las relaciones positivas y la comunicación entre los padres e hijos (6). Una escasa competencia parental, evidenciada por la permisividad, influye en el comportamiento del adolescente y puede conducirlo a conductas problemas (7). Entre los problemas sociales que se observan en la sociedad peruana están, el incremento del consumo de sustancias adictivas y la delincuencia juvenil, estos podrían tener menor incidencia si existieran niveles más altos de control parental (8). El reto de la función parental implica lograr satisfacer las diversas necesidades de los hijos (alimentación, cuidados corporales, protección, necesidades cognitivas, emocionales y socioculturales), pero considerando que las necesidades son evolutivas, los padres deben tener flexibilidad estructural que permita adaptarse a los cambios (9). Los cambios en las funciones de la familia y los cambios sociales generan nuevas necesidades, incluyendo demandas de aprendizaje de tareas para cubrir las diferentes etapas de la vida individual, familiar y social (10). Estos cambios requieren de la atención del profesional de enfermería; la acción de la enfermera familiar y comunitaria está orientada por principios y fundamentos científicos, humanísticos y éticos de respeto a la vida y a la dignidad humana, su práctica requiere de un abordaje interdisciplinario, de una actuación multiprofesional y en equipo, de una participación activa de las personas a las se atiende, con una visión holística del ser humano en su relación con el contexto familiar y social (11).

En la sociedad peruana existen diferentes niveles socioeconómicos, culturales y tipos de familia, aparentemente, la mayoría de ellas presentan habilidades y capacidades parentales deficientes, esto podría explicar la existencia de conductas problema en la adolescencia, ya que esta es una etapa crítica en el proceso del desarrollo. Es una tarea de la enfermera profesional atender las necesidades y preocupaciones de la familia en materia de salud, encontrando las potencialidades internas de la familia se busca reorientar su utilización o la identificación de los recursos externos que se encaminen a solucionarles sus necesidades promoviendo entornos saludables y orientarlos, de manera óptima y eficiente, a utilizar los servicios de salud disponibles. 
En la actualidad, los niños y adolescentes presentan diferentes necesidades en materia de salud, que no pueden ser atendidas por padres o profesores sin la ayuda de un profesional de salud. Diversos estudios demuestran el papel fundamental que cumple la enfermera escolar, la función desempeña en la administración de cuidados de salud; apoyo emocional a alumnos, profesores y padres; promueve un ambiente seguro, abogando por una escuela sin violencia y libre de problemas sociales; planifica, supervisa y realiza el seguimiento nutricional; coordina con los servicios de salud y gestión de temas sanitarios. En las instituciones educativas que existe un profesional de enfermería el estrés se reduce entre los profesores, lo cual mejora la calidad de la enseñanza y reduce el absentismo laboral, la enfermera escolar es el eje principal en la salud de la comunidad escolar; sin embargo, es una figura poco reconocida en nuestro país (12).

La aplicación del instrumento escala competencia parental percibida versión padres, ofrecerá un medio de aproximación diagnóstica para identificar a los padres con adecuada (positiva) o deficiente competencia parental, para su pronta actuación; esto permitirá entender cómo este proceso influye en la formación de la personalidad de sus hijos y conductas reflejadas en los comportamientos futuros; de esta manera, la disciplina de Enfermería en el campo comunitario contribuirá al pleno desarrollo y máximo bienestar físico, mental y social de toda la comunidad educativa, mediante la promoción de la salud y prevención de las enfermedades, por tal razón, se planteó como objetivo de investigación el determinar la validez del instrumento «escala de competencia parental percibida versión padres (ECPP-p)» para medir la competencia parental en el cuidado de los hijos en padres de escolares de $1 .^{\circ}$ al $6 .^{\circ}$ grado de primaria.

\section{MATERIAL Y MÉTODOS}

Estudio descriptivo de corte transversal, se utilizó estadística descriptiva, análisis factorial y pruebas para analizar la validez de la escala. Se llevó a cabo en la Institución Educativa Nacional «Maestro Ugo de Censi N. ${ }^{\circ}$ 86339» Chacas, Asunción - Ancash- Perú. Se aplicó el cuestionario a los padres cuyos hijos cursaban el $1 .^{\circ}$ al $6 .^{\circ}$ grado de primaria. La población estuvo conformada por 420 padres, la estructura de la escala competencia parental percibida en su versión para padres (ECPP-p) elaborado por Bayot, De Julián y Hernández, en versión en español, permite evaluar cinco dimensiones que se encuadran dentro del constructo «competencia parental»: implicación escolar, dedicación personal, ocio compartido, asesoramiento y orientación, y la asunción del rol de ser padre o madre. Se evalúa mediante 22 preguntas y siguiendo la escala tipo Likert de cuatro alternativas de respuesta en la que 1 es nunca, 2 es a veces, 3 es casi siempre y 4 siempre; las dos primeras dimensiones tienen como puntaje máximo 20 y un mínimo de 4, ya que constan de cinco preguntas cada una; en las tres dimensiones restantes, el puntaje más alto llega a ser 16 y el mínimo es 4 .

El instrumento fue sometido al juicio de expertos para analizar la validez de contenido, aplicando la prueba binomial a los resultados de los validadores. Al finalizar este proceso se invitó a los padres de familia a participar en las encuestas. Se aplicaron 500 de las cuales respondieron correctamente 420 . Las encuestas fueron codificadas para lograr el control de calidad, luego se ingresaron a una base de datos en el programa de Excel; posteriormente, esta base se transfirió al programa SPSS (Statistical Product and Service Solutions) versión 15.0 para el análisis correspondiente.

Se efectuó un análisis factorial confirmatorio con el programa AMOS 20.0 (analysis of moment structures); el procedimiento de ajuste del modelo de máxima verosimilitud se analizó a través del coeficiente de Mardia; para evaluar la bondad de ajuste absoluto se realizó la prueba chi cuadrado $\left(\mathrm{X}^{2}\right)$, la razón entre $\mathrm{X}^{2}$ y el número de grados de libertad, raíz del residuo cuadrático promedio de aproximación (RMSEA). Para probar la estructura factorial exploratoria se aplicó el método de los componentes principales, utilizando el KMO (Kaiser Meyer Olkin), prueba de Bartlett y rotación Varimax. La validez discriminante es la capacidad del instrumento para distinguir entre individuos o poblaciones que se espera que sean diferentes, evita la confusión y facilita la interpretación de las relaciones entre los constructos. Esta validez de la escala se contrastó por dos vías, matriz de correlaciones e intervalos de confianza al 95\%. Además, se calculó el coeficiente alfa de Cronbach de cada dimensión para determinar la confiabilidad.

Luego del análisis factorial por medio del programa SPSS se obtuvo tres componentes en la solución final, la Escala Competencia Parental Percibida versión padres (ECPPp) continúa constituida por 22 ítems englobados en tres dimensiones, dimensión 1: asunción del rol y dedicación personal de los padres conformada por 11 ítems (1,3,5,9,10,12,16,17,18,20,22 ); dimensión 2: implicación escolar integrada por 5 ítems $(4,11,13,15,21)$, y la dimensión 3: orientación y ocio compartido formada por 6 ítems $(2,6,7,8,14,19)$. Al aplicar el nuevo modelo se evaluaron las dimensiones con la siguiente puntuación: la primera dimensión tuvo un puntaje mínimo de 11 y un máximo de 
44; la dimensión 2 un mínimo de 5 y un máximo de 20 puntos, y en la tercera dimensión el puntaje mínimo fue de 6 y máximo 24. El puntaje de máximos y mínimos se obtuvo según la escala tipo Likert de cuatro alternativas de respuesta en la que 1 es nunca, 2 es a veces, 3 es casi siempre y 4 es siempre.

Para identificar la competencia parental adecuada o deficiente fue necesario atribuir indicadores (adecuada, regular y deficiente) a cada dimensión; por lo tanto, la dimensión « asunción del rol» y «dedicación personal de los padres» es deficiente cuando el puntaje obtenido se encuentra entre 11 - 22; regular entre 23 - 33 y adecuada entre 34 - 44 puntos; la dimensión «implicación escolar» resulta adecuada cuando el puntaje obtenido es de 16 y 20 puntos, regular entre 11 - 15 y deficiente entre 5 - 10 y la dimensión «orientación y ocio compartido» es deficiente si el nivel de puntuación se encuentra entre 6 - 12, regular entre 13 - 18 y adecuada entre 19 - 24 puntos.

\section{RESULTADOS}

La tabla 1 muestra la matriz de componentes rotados por el método de rotación Varimax con Káiser. El análisis estadístico del método de extracción agrupó los ítems en tres dimensiones, el cual fue analizado con pruebas estadísticas (ajuste absoluto, validez discriminante y confiabilidad).

En la tabla 2 la validez discriminante muestra correlación significativa, moderada y positiva, ninguno de los intervalos de confianza de estas correlaciones incluye a la unidad.

La tabla 3 el modelo propuesto se evaluó la confiabilidad a través del estadístico coeficiente alfa de Cronbach, se encontró un valor alto, lo que indica que la escala competencia parental percibida versión padres presenta alta confiabilidad. El resultado del coeficiente alfa de Cronbach deberá encontrarse entre los valores 0,70 y 0,90 para evidenciar confiabilidad del instrumento.

Tabla 1. Dimensiones de la escala competencia parental percibida en su versión padres en el mes de enero - 2014

\begin{tabular}{|c|c|c|c|}
\hline \multirow[t]{2}{*}{ Ítems } & \multicolumn{3}{|c|}{$\begin{array}{l}\text { Carga factorial de las } \\
\text { dimensiones }\end{array}$} \\
\hline & D1 & D2 & D3 \\
\hline \multicolumn{4}{|l|}{ D1 = Asunción del rol y dedicación personal de los padres } \\
\hline (10) Ayudo a mis hijos a establecer una rutina diaria en cuanto a hábitos de higiene. & 0,746 & 0,129 & 0,033 \\
\hline (9) Colaboro en las tareas del hogar. & 0,699 & 0,167 & 0,111 \\
\hline (1) Felicito a mis hijos cada vez que hacen algo bien. & 0,636 & 0,028 & 0,329 \\
\hline (17) Dedico un tiempo al día para hablar con mis hijos. & 0,593 & 0,187 & 0,296 \\
\hline (18) Ayudo a mis hijos a establecer una rutina diaria en lo referido al estudio. & 0,587 & 0,246 & 0,281 \\
\hline (5) Conozco los deberes y las tareas escolares que tienen mis hijos. & 0,567 & 0,327 & 0,180 \\
\hline (16) Existe una hora fija en la que mis hijos tengan que estar acostados o levantados. & 0,561 & 0,092 & 0,234 \\
\hline $\begin{array}{l}\text { (22) Soy muy consciente del cambio que ha experimentado mi familia con la llegada de } \\
\text { mis hijos. }\end{array}$ & 0,545 & 0,198 & 0,182 \\
\hline (20) Dispongo de suficiente tiempo para atender a mis hijos. & 0,463 & 0,433 & 0,158 \\
\hline (3) En casa, fomento que cada uno de los miembros de la familia exprese sus opiniones. & 0,449 & 0,166 & 0,389 \\
\hline $\begin{array}{l}\text { (12) Mantengo organizado una especie de archivo de mis hijos, donde se incluyan: datos } \\
\text { médicos, escolares, fotos, documentación legal, etc. }\end{array}$ & 0,384 & 0,306 & 0,301 \\
\hline \multicolumn{4}{|l|}{ D2 = Implicación escolar } \\
\hline (15) Establezco conjuntamente con el maestro, las metas y expectativas del niño. & 0,119 & 0,706 & 0,208 \\
\hline $\begin{array}{l}\text { (11) Mantengo un contacto frecuente con la escuela para estar al tanto de cualquier } \\
\text { información referente a los programas y recursos para las familias. }\end{array}$ & 0,215 & 0,669 & 0,143 \\
\hline (4) Consulto con el profesor sobre la evolución de mis hijos en el colegio. & 0,101 & 0,663 & 0,230 \\
\hline (13) Tengo una mentalidad positiva y abierta al conversar con el personal de la escuela. & 0,210 & 0,617 & 0,186 \\
\hline $\begin{array}{l}\text { (21) Asisto regularmente a las reuniones que se establecen en la asociación de padres de } \\
\text { alumnos. }\end{array}$ & 0,525 & 0,541 & $-0,136$ \\
\hline
\end{tabular}


D3= Orientación y ocio compartido.

(6) Frecuento lugares donde hay más niños para potenciar que mis hijos se relacionen.

$\begin{array}{lll}0,094 & 0,302 & 0,659 \\ 0,323 & 0,010 & 0,621 \\ 0,000 & 0,394 & 0,554 \\ 0,264 & 0,244 & 0,500 \\ 0,391 & 0,186 & 0,483 \\ 0,419 & 0,060 & 0,446\end{array}$

(7) Veo con mis hijos, ciertos programas de TV y los comento con ellos después.

(19) Hago pequeñas excursiones con la familia al cine, zoológico, museos, parques, etc.

(14) Respondo a las encuestas escolares que busquen averiguar los intereses, talento y habilidades de mis hijos.

(2) Refuerzo en casa las reglas, normas y expectativas de conducta de la escuela.

0,419

(8) Me preocupo de incluir a mis hijos en actividades extraescolares.

Tabla 2. Validez discriminante según matriz de correlaciones de la escala competencia parental percibida en su versión padres, para tres dimensiones en el mes de enero - 2014

DIM 2

DIM 3

\begin{tabular}{|c|c|c|c|c|c|}
\hline & \multicolumn{3}{|c|}{ Correlación de Pearson } & $0,621^{* *}$ & $0,681^{* *}$ \\
\hline & \multicolumn{3}{|c|}{ Sig. (bilateral) } & 0,000 & 0,000 \\
\hline & \multicolumn{3}{|l|}{$\mathrm{N}$} & 420,000 & 420,000 \\
\hline \multirow[t]{7}{*}{ DIM 1} & \multirow{4}{*}{ Bootstrap $^{b}$} & \multicolumn{2}{|l|}{ Sesgo } & 0,000 & 0,000 \\
\hline & & \multicolumn{2}{|l|}{ Tipo Error } & 0,032 & 0,028 \\
\hline & & \multirow{2}{*}{$\begin{array}{l}\text { Intervalo de confianza al } \\
95 \%\end{array}$} & Inferior & 0,556 & 0,620 \\
\hline & & & Superior & 0,679 & 0,732 \\
\hline & \multicolumn{2}{|c|}{ Correlación de Pearson } & & & $0,558^{* *}$ \\
\hline & \multicolumn{2}{|c|}{ Sig. (bilateral) } & & & 0,000 \\
\hline & \multicolumn{2}{|l|}{$\mathrm{N}$} & & & 420,000 \\
\hline \multirow[t]{4}{*}{ DIM 2} & \multirow{4}{*}{ Bootstrap ${ }^{b}$} & Sesgo & & & 0,000 \\
\hline & & Tipo Error & & & 0,036 \\
\hline & & \multirow{2}{*}{$\begin{array}{l}\text { Intervalo de confianza al } \\
95 \%\end{array}$} & Inferior & & 0,484 \\
\hline & & & Superior & & 0,629 \\
\hline
\end{tabular}

D= Dimensión; $N=$ =Población; $D 1$ = Asunción del rol y dedicación personal de los padres; D2=Implicación escolar; D3= Orientación y ocio compartido, Sig=Nivel de correlación, Bootstrap=Procedimiento del programa SPSS.

Tabla 3. Nivel de confiabilidad de la escala competencia parental percibida versión padres para cinco y tres dimensiones en el mes de enero - 2014

\begin{tabular}{lc}
\hline \multicolumn{1}{c}{ Dimensiones } & Alfa de Cronbach \\
\hline D1, Implicación escolar 5 ítems. & 0,749 \\
D2, Dedicación personal 5 ítems. & 0,752 \\
D3, Ocio compartido 4 ítems. & 0,624 \\
D4, Asesoramiento y la orientación 4 ítems. & 0,712 \\
D5, Asunción del rol de ser padre/madre 4 ítems. & 0,697 \\
Total 5 dimensiones, 22 ítems & 0,870 \\
\hline
\end{tabular}




\begin{tabular}{lc}
\hline \multicolumn{1}{c}{ Dimensiones } & Alfa de Cronbach \\
\hline D1, Asunción del rol y dedicación personal de los padres 11 ítems. & 0,865 \\
D2, Implicación escolar 5 ítems. & 0,749 \\
D3, Orientación y ocio compartido 6 ítems. & 0,722 \\
Total 3 dimensiones, 22 ítems & 0,769 \\
\hline
\end{tabular}

\section{DISCUSIÓN}

En este estudio se validó el cuestionario ECPP-p el cual cumple con propiedades psicométricas adecuadas y presenta validez de contenido lo que permitió continuar con el estudio ya que el instrumento de medición representa a todos los ítems del dominio de contenido de la variable medida. La validez de constructo es el grado en que el instrumento representa y mide un concepto teórico, se puede verificar a través del análisis factorial, este evidencia la relación entre la conceptualización teórica del instrumento y su estructura factorial. Existen dos tipos de análisis factorial: exploratorio y confirmatorio; el análisis factorial exploratorio es un conjunto de métodos estadísticos multivariados de interdependencia, cuyo propósito es identificar una estructura de factores subyacentes a un conjunto amplio de datos (13), en el cuestionario se aplicó el método de rotación ortogonal Varimax con Káiser, este método minimiza el número de variables que tienen saturaciones altas en cada factor (2).

A partir del análisis de la validez de constructo de los 22 ítems de la escala de Competencia Parental Percibida, se obtuvo tres dimensiones según su comportamiento en la solución final. Esta estructura difiere con el modelo hipotético utilizado en el estudio de Bayot (2005) que, a través del mismo método, obtuvo cinco dimensiones que explicaron el 48,80\% de la varianza total. Con el resultado de este estudio se analizó la rotación de los componentes, se consideró la agrupación de los ítems y se realizó ajustes en la denominación de las dimensiones. La confiabilidad fue evaluada mediante la consistencia interna, que es el grado en que los ítems de una escala se correlacionan entre sí (14). La ECPP-p es confiable ya que los datos obtenidos confirman que la consistencia interna del cuestionario es alta $(a=0,87)$, contrastado con el resultado mostrado en el estudio realizado por Bayot $(a=0,86)$, a pesar que la escala se aplica en diferente área de estudio (15).

Con relación a las dimensiones, no hay mayor relevancia en la dimensión implicación escolar (alfa $=0,76$ / 0,75), a diferencia de las demás dimensiones. En este estudio se encontró que en la dimensión ocio compartido $(0,62)$ el alfa de Cronbach obtiene el menor de todas las dimensiones; sin embargo, en la clasificación de Alfa de Cronbach de Hernandez Sampiere et al. (2006), el rango de confiabilidad aceptable varía entre 0,6 a 0,8 (16). En el estudio realizado por Bayot se obtuvo cuatro dimensiones cuyos valores se encuentran por debajo del rango aceptable. La dimensión dedicación personal presentó un alfa de Cronbach igual a 0,68 , a diferencia de este estudio cuyo alfa de Cronbach fue 0,75 ; la dimensión «asesoramiento/ orientación» mostró alfa igual a 0,62 y, en este estudio, fue a $=0,7$; la «asunción del rol de ser padre/madre» tuvo un alfa igual a 0,52 discrepando con este estudio que resultó 0,70 y, finalmente, la dimensión «ocio compartido» tuvo un alfa de Cronbach igual a 0,56. En el modelo propuesto, la confiabilidad de cada dimensión es mayor en comparación al de cinco dimensiones; sin embargo, la confiablidad total es más alta en el modelo original.

La validez discriminante es la capacidad del instrumento para distinguir entre individuos o poblaciones que se espera que sean diferentes; se realiza a través de los siguientes métodos: comparación entre las correlaciones de los indicadores; comparación entre la varianza compartida y la varianza extraída, y el intervalo de confianza entre las correlaciones (17). Un intervalo de confianza permite verificar hipótesis planteadas acerca de parámetros poblacionales, esta aporta más información que un estimador puntual. Por tanto, según este criterio, el estudio presenta una adecuada validez discriminante; asimismo, cumple con los criterios de normalidad, ya que los ítems presentan adecuada distribución y distribución multivariada. Estos resultados no difieren del estudio realizado por Bayot, quien encontró adecuada validez discriminante y normalidad multivariada; sin embargo, el autor utilizó este análisis para encontrar diferencias entre las características de estado civil, profesión y los estudios cursados respecto a cada dimensión de la ECPP-p (18).

Se afirma que la Escala es confiable y válida; no obstante, se sabe que todo estudio de investigación posee debilidades y fortalezas. Una de las debilidades del estudio realizado es que el nuevo modelo presenta un alfa de Cronbach 
menor en relación al de cinco dimensiones. Al evaluar cada dimensión en el modelo original, dos de ellas presentan valores de alfa de Cronbah inferiores a 0,70; mientras que las tres dimensiones del nuevo modelo poseen alfa de cronbach mayor a 0,7 ; sin embargo, ambos modelos resultan confiables, siendo esta una fortaleza del estudio, ya que la escala de competencia parental percibida versión padres (ECPP-p) mide el constructo «competencia parental». Otra fortaleza de este estudio es la aplicación de la escala en el Perú ya que es confiable y válida para evaluar el tipo de competencia parental. Los ítems del cuestionario son sencillos, claros y suficientes, esto facilita la compresión del lector, y el tiempo empleado para desarrollar el cuestionario no requiere más de 15 minutos, todo esto garantiza la colaboración de los padres.

Los datos obtenidos en el estudio permiten afirmar que el instrumento es confiable y válido para medir competencia parental en nuestra realidad, considerando que fue aplicado por primera vez en el Perú, muestra alta confiabilidad y validez. Se sugiere utilizar la escala competencia parental percibida versión padres según el resultado de la presente investigación, como un modelo más adaptado a la realidad peruana. Aunque no se excluye el uso de la ECPP-p como originalmente fue planteada, además, se recomienda al profesional de enfermería comunitaria aplicar la escala para identificar el tipo de competencia parental que desarrollan los padres para trabajar en la promoción del bienestar familiar.

\section{REFERENCIAS BIBLIOGRÁFICAS}

1. Argibay J. Técnicas psicométricas cuestiones de validez y confiabilidad. UBA- UCES 2006; 15- 33.

2. Análisis Factorial [en línea] [citado 20 mar 2014]; URL disponible en: http://halweb.uc3m.es/esp/Personal/ personas/jmmarin/esp/AMult/tema4am.pdf.

3. Torío López S, Peña Calvo J, Rodríguez Menéndez M. Estilos educativos parentales, revisión bibliográfica y reformulación teórica. BIBLID 2008; 20: 151-178.

4. Rodrigo López J, Martin Quintana J, Cabrera Casimiro E, Máiquez Chaves L. Las competencias parentales en contextos de riesgo psicosocial. Psychosocial Intervention; 18 (2): 113-120.

5. Estay Barrera F, Jara Males P, Mora San Martín M. Manual de apoyo para la Formación de Competencias Parentales, Programa Abriendo Caminos. Santiago de Chile: MIDEPLAN; 2009.

6. Sanhueza Díaz L. Desafíos y oportunidades en la evaluación y fortalecimiento de competencias parentales [Internet] 2010 [citado 05 julio 2012]; URL disponible en: http://www.rediberoamericana detrabajoconfamilias.org/ cancilleria03.pdf.

7. Cabanillas Rojas W. Factores de riesgo/protección y los programas preventivos en drogodependencias en el Perú. Rev. Perú. med. exp. salud publica 2012; 29(1):104-111.

8. Basanta Dopico J. Competencia parental y necesidades criminógenas en una muestra de menores infractores. IPSE-ds [Internet]. 2012; [citado 20 marzo 2013]: 5: 37-46. Disponible en: http:/ /www.webs.ulpgc.es/ipseds/w_IPSE-ds05a03.pdf.

9. Palacios Delgado J, Andrade Palos P. Influencia de las prácticas parentales en las conductas problema en adolescentes. Ciencias sociales y humanidades 2008; 7(7): 7-18.

10. Roque Cala K, Páez Rovira M, Cantillo Vento H. La familia como pilar básico para la educación. Mendive 2012 (40).

11. Pérez Giraldo B. El cuidado de enfermería en familia: un reto para el siglo XXI [en línea] 2009 [fecha de acceso 08 de noviembre de 2012]; URL disponible en: http://aquichan.unisabana.edu.co/index.php/ aquichan/article/view/19/37.

12. Heierle Valero C. Salud y Cuidados en la Familia. Index de Enfermería [Internet] 2001; [citado 16 marzo 2014]. Disponible en: http://www.index-f.com/ index-enfermeria/34revista/34_articulo_7-8.php.

13. Pérez Gil J, Chacón Moscoso S, Moreno Rodríguez R. Validez de constructo: el uso de análisis factorial exploratorio-confirmatorio para obtener evidencias de validez. Psicothema [Internet]. 2000, [citado $18 \mathrm{mar}$ 2013]: 12(2): 442-446. Disponible en http:// www.psicothema.com/pdf/601.pdf.

14. Campo Arias A, Oviedo H. Propiedades psicométricas de una escala: la consistencia interna. Revista de Salud Pública 2008, 10 (5): 831-839. Disponible en http://redalyc.uaemex.mx/src/inicio/ ArtPdfRed.jsp?iCve=42210515.

15. Bayot A, Hernández Viadel J, Felipe de Julián L. Análisis factorial exploratorio y propiedades psicométricas de la escala de competencia parental percibida. Versión para padres/madres (ECPP-p). Relieve 2005; 11(2): 113-126.

16. Hernández Sampiere R, Fernández Collado C, Baptista Lucio P. Metodología de la investigación.4ta ed. México: Mc Graw Hill; 2006.

17. Martínez García J, Martínez Caro L. La validez discriminante como criterio de evaluación de escalas: ¿teoría o estadística? Univ. Psychol (Colombia) 2009; 8 (1): 27-36.

18. Lamprea M, Gómez Restrepo. Validez en la evaluación de escalas. rev. colomb. psiquiatr. 
[Internet]. 2007 [citado 20 mar 2014]; 36(2): 340-348. Disponible en: http://www.scielo.org.co/pdf/rcp/ v36n2/v36n2a13.pdf

\section{Correspondencia}

Ceci VeraVásquez

Universidad Peruana Cayetano Heredia

Dirección: Av. Honorio Delgado 430, Urb. Ingeniería, S.M.P.

Correo electrónico: ceci.vera@upch.pe

Forma de citar este artículo: Vera-Vásquez C, Zaragoza-Tafur A R, Musayón-Oblitas F Y. Validación de la «escala de competencia parental percibida versión padres (ECPP-P)» para el cuidado de los hijos Institución Educativa Nacional «Maestro Ugo de Censi N. 86339» Chacas, Asunción - Ancash- Perú. Rev. enferm Herediana.2014;7(1):17-24. 